\title{
SAR Imaging Based on Compressive Sensing via Fractional Fourier Transform
}

\author{
Xiaolong $\mathrm{Li}^{1}$, Yunqing $\mathrm{Liu}^{1}$, Shuang $\mathrm{Zhao}^{1}$ and Wei $\mathrm{Chu}^{2}$ \\ ${ }^{1}$ School of Electronics and Information Engineering, Changchun University of Science and \\ Technology, Changchun 130022, China \\ ${ }^{2}$ School of Electronics and Information Engineering, Changchun University, Changchun 130022, \\ China \\ Email: k68b7@163.com; mzliuyunqing@163.com; star_billy@163.com; \\ chu13159648838@126.com
}

Keywords: CS; FRFT; SAR imaging.

\begin{abstract}
To reduce the amount of measurements, compressive sensing (CS) has been introduced to synthetic aperture radar (SAR). In this paper a novel synthetic aperture radar (SAR) imaging algorithm based on compressive sensing (CS) is proposed. In the range plane, the echo signal is reconstructed in fractional Fourier domain for the sparsity, and matched filtering is used in the azimuth to obtain the image. Taking into account the large peak signal to noise ratio (PSNR), raising compression ratio can enhance the resolution, improve the range ambiguity and possess benign focusing ability. Simulation results show that by using the method this paper proposed it outperforms the existing Range Doppler algorithm demonstrating the superior performance of the proposed approach.
\end{abstract}

\section{Introduction}

Synthetic Aperture Radar (SAR) is an active remote sensing system which is widely utilized in many fields such as civilian, military, and biomedical. The main advantages of SAR are that it can reduce the effects of clouds and fog and allow them to be independent of external sources for imaging, having day and night and all-weather imaging capability.

In these applications, a wide ground coverage and high azimuth resolution is always a primary preference for efficient use of the resources. Future SAR will be required to produce high-resolution image over a wide area of surveillance. However, in conventional SAR systems, high geometric resolution in azimuth and wide swath coverage imposes contradicting requirements on system design: a high azimuth resolution requires a large Doppler bandwidth, which has to be sampled with a sufficiently high pulse repetition frequency (PRF). Any deviation of PRF could produce nonuniform sampling, leading to serious azimuth ambiguity if a matched filter is used directly, so further processing of the received signals is required before conventional monostatic SAR algorithms are applied. However, such a reconstruction is not robust when the samples are approximately overlapped. Another defection in SAR system is that a sufficient sampling rate for range signal and a dense azimuth sampling are needed. The receivers will lead to huge data volume and bring a challenge not only to the analog-to-digital (A/D) converter, but also to the on-board storage [1-6].

The recently introduced theory of compressed sensing (CS) is a new concept allowing the recovery of sparse signal that has been sampled below the traditional Nyquist sampling rate. In CS framework, it uses a low-dimensional nonadaptive, linear projection to acquire an efficient representation of a sparse signal with just a few measurements. Due to its compressed sampling ability, compressed sensing has found many attractions in radar remote sensing and branched out to many new fronts. R. Baraniuk et al. proposed the radar imaging system based on CS for the first time and a high resolution imaging method is presented in for SAR sparse targets reconstruction based on CS theory [7-15]. In the above researches, it has been shown that a successful recovery of 
a compressible signal depends on the presence of sparse dictionary and these works have made a great contribution to the future research of radar signal processing.

In this paper, we introduce a novel synthetic aperture radar imaging algorithm which named CS-FRFT based on compressed sensing theory and fractional Fourier transform (FRFT) techniques. Our approach is in contrast to other traditional radar related algorithms that have considered using CS as part of one-dimensional analog-to-information conversion. The key idea in our approach is to use CS to reconstruct one-dimensional (1-D) target in the range dimension via FRFT. This radar system randomly transmits fewer pulses in azimuth plane and samples fewer data than traditional systems at random intervals in range plane. In addition, the proposed algorithm can reduce the required receiver analog-to-digital (A/D) conversion band-width so that it need operate only at the reflectivity's potentially low "information rate" rather than at its potentially high Nyquist rate. We present an extraordinary and innovative application of CS for SAR. 1) The algorithm achieves 2-D imaging of the targets via constructing the range measurement matrix using CS. 2) The CS-FRFT technology is used in this algorithm to resolve high resolution. This will directly impact A/D conversion, and has the potential to reduce the overall data rate and to simplify hardware design. Meanwhile, it provides the potential to achieve higher resolution between targets than traditional algorithms. The simulated results verify the validity of this paper.

\section{Compressive Sensing Theory}

The Shannon-Nyquist sampling theorem requires a signal to be sampled at a frequency of twice its bandwidth to be able to reconstruct it exactly. In CS framework, it uses a low-dimensional, nonadaptive, linear projection to acquire an efficient representation of a sparse signal with just a few measurements, so as to greatly reduce the sampling rate and enhance the data rate.

Let $\mathbf{f}$ represent the $N \times 1$ unknown signal, which is compressible in a linear basis $\psi$ (such as a wavelet basis). In other words, $\mathbf{f}=\boldsymbol{\psi} \mathbf{w}$, where $\mathbf{w}$ is an $N \times 1$ sparse signal, most of its coefficients are zero. Consider the following acquisition system:

$$
\mathbf{y}=\Phi \mathbf{f}+\mathbf{n}
$$

where $M \times 1$ linear measurements $\mathbf{y}$ of the original unknown signal $\mathbf{f}$ are taken with an $N \times N$ measurement matrix $\Phi^{\prime}=\left[\phi_{1}^{\prime}, \phi_{2}^{\prime}, \ldots, \phi_{N}^{\prime}\right]$ and represents the acquisition noise. We can also write Eq. (1) in terms of the sparse transform coefficients as:

$$
\mathbf{y}=\boldsymbol{\Phi} \mathbf{w}+\mathbf{n}
$$

where $\boldsymbol{\Phi}=\boldsymbol{\Phi} \boldsymbol{} \boldsymbol{\Psi}$, which is the commonly used notation in the CS literature and will be adopted in the rest of this paper.

According to the theory of compressive sensing, when the number of measurements is small compared to the number of signal coefficients $M \ll N$, under certain conditions the original signal $\mathbf{f}$ can be reconstructed very accurately by utilizing appropriate reconstruction algorithms.

Since the number of measurements $M$ is much smaller than the number of unknown coefficients $\mathbf{w}$, the original signal cannot be obtained directly from the measurements. The inversion of (1) or (2) is required, which is an ill-posed problem. Therefore, compressive sensing incorporates a reconstruction mechanism to obtain the original signal. By exploiting the sparsity of $\mathbf{w}$, the inverse problem is regularized constraining the $l_{0}$ norm of $\mathbf{w},\|\mathbf{w}\|_{0}$, which is equal to the number of nonzero terms in w. An approximation to the original signal is then obtained by solving the following optimization problem:

$$
\hat{\mathbf{w}}=\underset{\mathbf{w}}{\arg \min }\left\{\|\mathbf{y}-\boldsymbol{\Phi} \mathbf{w}\|_{2}^{2}+\tau\|\mathbf{w}\|_{0}\right\}
$$

This optimization problem is NP-hard; therefore, some simplifications are used. The most common one is to use the $l_{1}$-norm instead of the $l_{0}$-norm, so that the optimization problem becomes

$$
\hat{\mathbf{w}}=\underset{\mathbf{w}}{\arg \min }\left\{\|\mathbf{y}-\boldsymbol{\Phi} \mathbf{w}\|_{2}^{2}+\tau\|\mathbf{w}\|_{1}\right\}
$$

A number of methods have been proposed to solve the CS reconstruction problems defined in (3) 
and (4) or their extensions (for example, formulations utilizing $l_{p}$ norms for $\mathbf{w}$ with $0<p<1$ ). Most of the proposed methods are examples of energy minimization methods, including linear programming algorithms and constructive (greedy) algorithms. Additionally, sparse signal representation is a very close topic to CS, and many algorithms proposed there can also be applied to the CS reconstruction problem.

\section{Fractional Fourier Transform On SAR Imaging}

\section{A. Baseband Signal Model}

A typical radar linear frequency modulation (LFM) waveform can be expressed by:

$$
s(t)=\operatorname{rect}\left(t / T_{p}\right) \cos \left(2 \pi f_{0} t+K \pi t^{2}\right)
$$

where $f_{0}$ represents the carrier frequency, $K$ represents the slope of linear frequency modulation; $T_{p}$ is the width of pulse. The echo signal of scattering center can be expressed by:

$$
s_{r}(t)=\sum_{n=1}^{N} \sigma_{n} r e c t\left(\frac{t-2 R_{n} / c}{T_{p}}\right) \times \cos \left(2 \pi f_{0}\left(t-\frac{2 R_{n}}{c}\right)+\frac{1}{2} K\left(t-\frac{2 R_{n}}{c}\right)^{2}\right)
$$

where $N$ is the number of the scattering points, $\sigma_{n}$ is the radar cross section, rect $(t)$ is the envelope of radar pulse, $c$ is the velocity of electromagnetic wave, $R_{n}$ is the distance between radar and scattering point. And with the expression $h(t)=\exp \left(-j 2 \pi f_{0} t\right)$, we can calculate the baseband echo signal. It is expressed by:

$$
s_{B}(t)=\sum_{n=1}^{N} \sigma_{n} r e c t\left(\frac{t-2 R_{n} / c}{T_{p}}\right) \exp \left(j\left(-4 \pi f_{0} R_{n} / c\right)\right) \exp \left(j\left(\frac{1}{2} K\left(t-2 R_{n} / c\right)^{2}\right)\right)
$$

With $\alpha_{n}=\sigma_{n} \exp \left(j\left(-4 \pi f_{0} R_{n} / c\right)\right)$, the equation can be rewritten as:

$$
s_{B}(t)=\sum_{n=1}^{N} \alpha_{n} r e c t\left(\frac{t-2 R_{n} / c}{T_{p}}\right) \exp \left(j\left(\frac{1}{2} K\left(t-2 R_{n} / c\right)^{2}\right)\right)
$$

\section{B. Range Compression on CS with FRFT}

The fractional Fourier transform, which is a generalization of the ordinary Fourier transform, can be considered as a rotation by an angle $\alpha$ (not multiple of $\pi / 2$ ) in the time-frequency plane or a decomposition of the signal in terms of chirps. It also serves as an orthonormal signal representation for chirp signals. The fractional Fourier transform is also called rotational Fourier transform or angular Fourier transform. The fractional Fourier transform is computed using the angle of rotation in the time-frequency plane as the fractional power of the ordinary Fourier transform. Let $x(u)$ be an arbitrary signal, its $p^{\text {th }}$-order FRFT is defined as:

$$
X_{\alpha}(u)=\int K_{\alpha}(u, t) x(t) d t
$$

where, $p$ presents the fractional transformation order (corresponding to a rotation angle $\alpha=p \pi / 2$ with $p \in \mathbf{R})$.

$K_{\alpha}(u, t)$ represents the FRFT kernel function and is defined as:

$$
\begin{gathered}
K_{\alpha}(u, t)=\left\{\begin{array}{l}
A_{\alpha} \cdot \exp \left[j \pi\left(\begin{array}{l}
\left(u^{2}+t^{2}\right) \cot \alpha \\
-2 u t \csc \alpha
\end{array}\right)\right] \\
\alpha \neq n \pi \\
\delta(u-t) \quad \alpha=2 n \pi \\
\delta(u-t) \quad \alpha=(2 n \pm 1) \pi
\end{array}\right. \\
A_{\alpha}=\sqrt{1-j \cot \alpha / 2 \pi}
\end{gathered}
$$

The fractional Fourier transform has the ability to process chirp signals better than the ordinary Fourier transform. This is because a chirp signal forms a line in the time-frequency plane, and therefore, there exists an order of transformation in which such signals are compact. Chirp signals are not compact in the time or spatial domain. Thus we can extract the signal easily in an appropriate fractional Fourier domain when it is not possible to separate the signal and noise in spatial or 
frequency domain.

The atom dictionary $\mathbf{D}$ can be constructed by the following two steps.

First step: construct the $\mathbf{a}_{1}, \mathbf{a}_{2}$ vector with $\alpha$, the element of vector should satisfy:

$$
\begin{gathered}
\mathbf{a}_{1}(n)=\exp \left(j\left((\cot \alpha)\left(n-1-\frac{N}{2}\right)^{2} \frac{\beta^{2}}{2}\right)\right) n=1,2,3 \cdots N \\
\mathbf{a}_{2}(n)=\exp \left(j\left((\cot \alpha)(n-1)^{2} \frac{T_{s}^{2}}{2}\right)\right) n=1,2,3 \cdots N \\
\beta=2 \pi \sin \alpha /\left(N^{*} T_{s}\right)
\end{gathered}
$$

here $\alpha$ is the angle of rotation, $T_{s}, N$ represent sampling time and sampling points respectively.

Second step: Construct diagonal matrix $\mathbf{A}_{1}$ and $\mathbf{A}_{2}$ with $\mathbf{a}_{1}, \mathbf{a}_{2}$, they are defined as:

$$
\begin{aligned}
\mathbf{A}_{1} & =\left[\begin{array}{ccccccc}
a_{1}(1) & 0 & 0 & 0 & 0 & \cdots & 0 \\
0 & a_{1}(2) & 0 & 0 & 0 & \cdots & 0 \\
0 & 0 & a_{1}(3) & 0 & 0 & \cdots & 0 \\
\vdots & & \ddots & & & \vdots \\
0 & 0 & \cdots & a_{1}(N-1) & 0 \\
0 & 0 & 0 & \cdots & a_{1}(N)
\end{array}\right]_{N \times N} \\
\mathbf{A}_{2} & =\left[\begin{array}{ccccccc}
a_{2}(1) & 0 & 0 & 0 & 0 & \cdots & 0 \\
0 & a_{2}(2) & 0 & 0 & 0 & \cdots & 0 \\
0 & 0 & a_{2}(3) & 0 & 0 & \cdots & 0 \\
\vdots & & \ddots & & & \vdots \\
0 & 0 & \cdots & a_{2}(N-1) & 0 \\
0 & 0 & 0 & \cdots & a_{2}(N)
\end{array}\right]_{N \times N}
\end{aligned}
$$

So, the dictionary is $\mathbf{D}=\mathbf{A}_{1} \mathbf{W} \mathbf{A}_{2}$

Matrix of coefficients is given by $\mathbf{W}=\frac{\mathrm{FFT}(\mathbf{E})}{\sqrt{N}}$ where $\mathbf{E}$ is $N \times N$ dimensions unit matrix, FFT represents fast Fourier transform. Finally we can conclude the observational matrix.

Then, according to CS theory, we can get the signal which is compressed in range plane. After compression, with the results, the SAR image can be obtained by matched filter in azimuth plane.

\section{Azimuth Compression}

After the signal processed in range plane, the signal in azimuth plane is also a chirp signal, and the reference function of matched filter in azimuth plane can be expressed by:

$$
H_{a}\left(f_{\eta}\right)=\exp \left(-j \pi \frac{f_{\eta}^{2}}{K_{a}}\right)
$$

Where $K_{a}=-\frac{2 V^{2}}{\lambda R_{0}}$ is the chirp rate of Doppler frequency modulation, for the purpose of azimuth compression, the signal processed with CS theory in range plane multiplied by $H_{a}\left(f_{\eta}\right)$, the result is as follows:

$$
S_{3}\left(\tau, f_{\eta}\right)=S_{2}\left(\tau, f_{\eta}\right) H_{a}\left(f_{\eta}\right)=A_{0} p_{r}\left(\tau-2 R_{0} / c\right) W_{a}\left(f_{\eta}-f_{\eta_{c}}\right) \times \exp \left(-j \frac{4 \pi f_{0} R_{0}}{c}\right)
$$

And $\mathrm{s}_{a c}(\tau, \eta)=\operatorname{IFFT}_{\eta}\left\{S_{3}\left(\tau, f_{\eta}\right)\right\}$ is the image we need, where IFFT represents inverse fast Fourier transform.

\section{Simulation Results}

According to the simulation results, this paper verifies the validity of SAR imaging based on compressive sensing theory via fractional Fourier transform technology. The simulated parameters of point target are as followed: carrier frequency is $10 \mathrm{GHz}$, sampling rate is $600 \mathrm{~Hz}$, velocity of aircraft is $200 \mathrm{~m} / \mathrm{s}$, bandwidth is $200 \mathrm{MHz}$. Fig. 1 (a) and Fig. 1 (b) are the image with the fixed resolution, Fig. 
1 (a) represents imaging of three point targets using CS recovery and Fig. 1(b) represents imaging of three point targets by range Doppler algorithm. Compared with Fig. 1 (b) it is clear to identify three point targets in Fig. 1 (a). The method of CS recovery can improve the resolution. And Fig. 2 represents the three-dimensional imaging of the two algorithms.

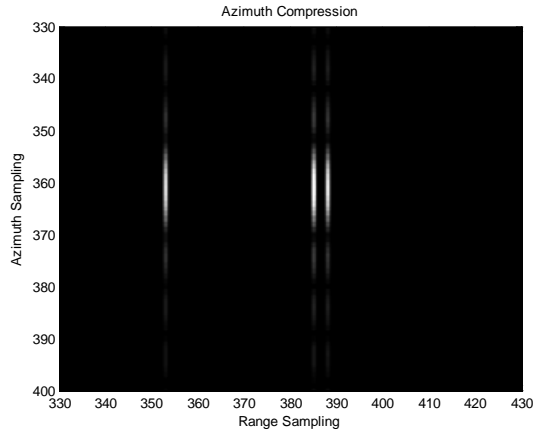

(a)

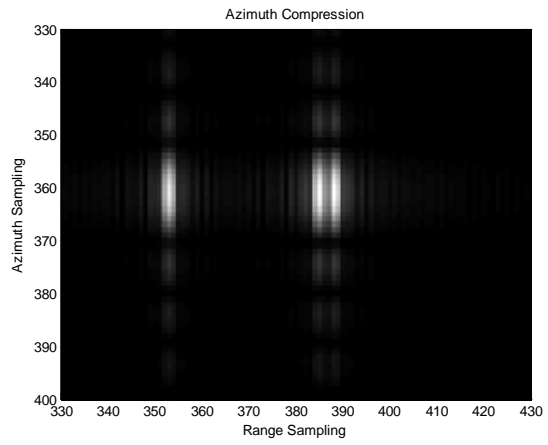

(b)

Figure 1. Comparison of reconstruction results: (a) CS algorithm with FRFT. (b) Traditional RD algorithm

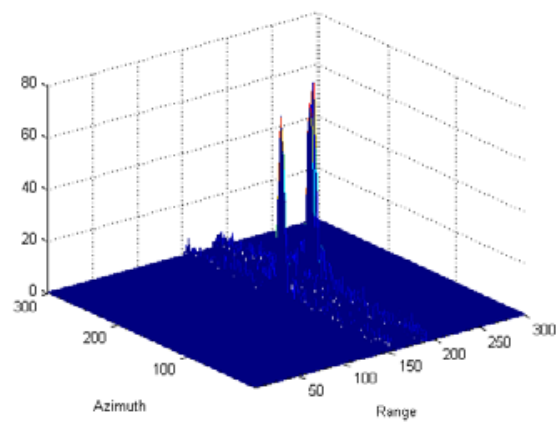

(a)

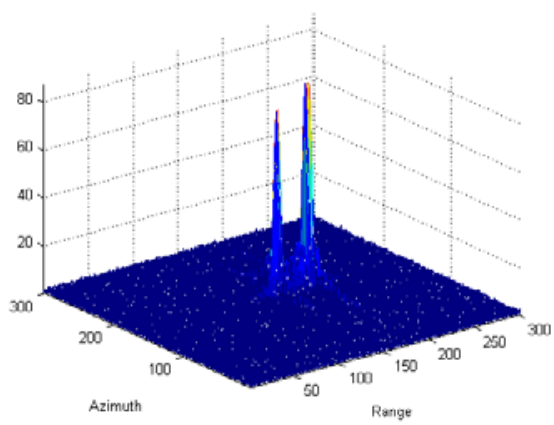

(b)

Figure 2. Comparison of reconstruction results: (a) CS algorithm with FRFT. (b) RD algorithm

PSNR is an engineering term for the ratio between the maximum possible power of a signal and the power of corrupting noise that affects the fidelity of its representation. Because many signals have a very wide dynamic range, PSNR is usually expressed in terms of the logarithmic decibel scale. PSNR is most easily defined via the mean squared error (MSE). Given a noise-free $m \times n$ monochrome image $I$ and its noisy approximation $K$, MSE is defined as:

$$
M S E=\frac{1}{m \times n} \sum_{i=0}^{n-1} \sum_{j=0}^{m-1}|I(i, j)-K(i, j)|^{2}
$$

and PSNR is defined as:

$$
P S N R=10 \times \log \left(\frac{255^{2}}{M S E}\right)
$$

where 255 is the maximum gray value of 8 bits binary gray image, $m \times n$ is the size of image, $(i, j)$ denotes coordinate.

By means of MSE and PSNR, the quality of image is analyzed as Fig. 4 described in detail.

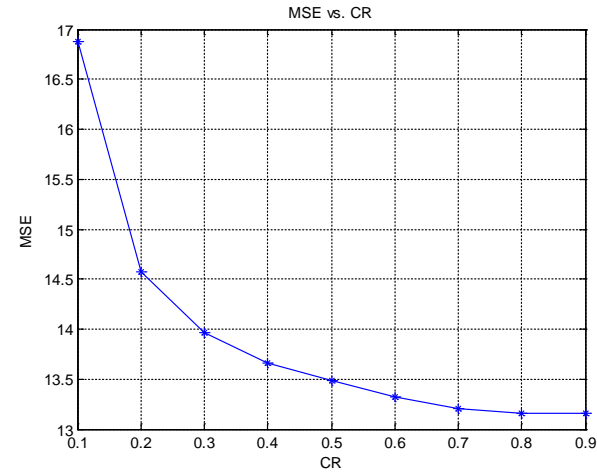

(a)

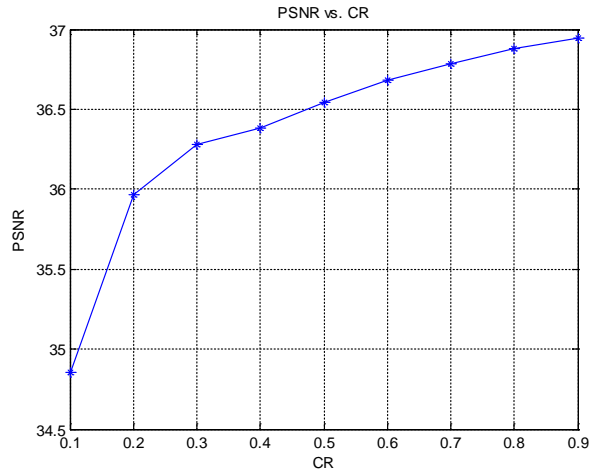

(b)

Figure 3. The results of performance analysis with SNR $=0 \mathrm{~dB}$ : (a) MSE for different CR, (b) PSNR for different CR. 


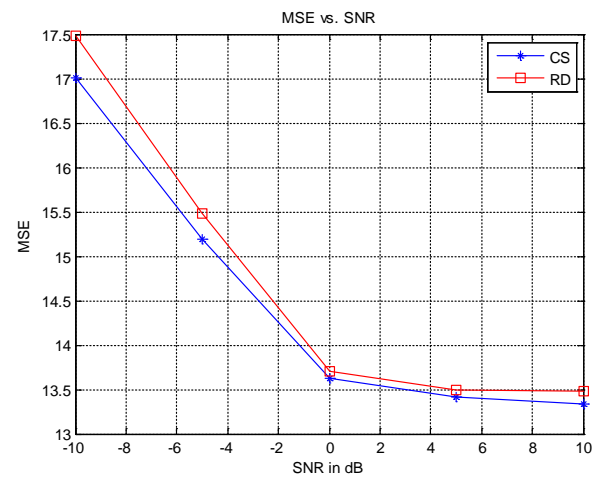

(a)

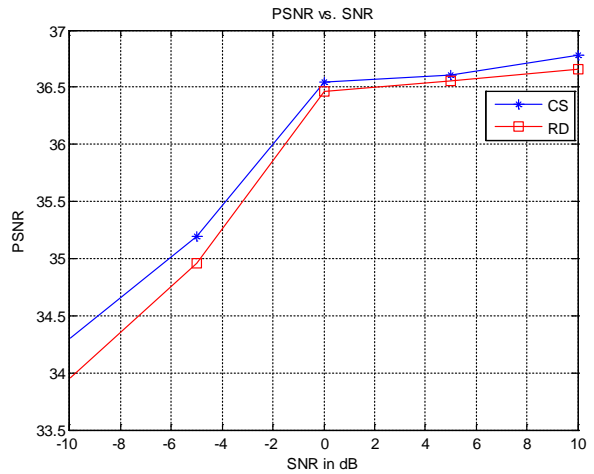

(b)

Figure 4. Results of performance analysis with CR = 0.5: (a) MSE vs. SNR, (b) PSNR vs. SNR.

In Fig. 3 with CR increasing, the value of MSE is getting smaller, and the value of PSNR is getting bigger. When CR is 0.3, the targets can be identified with the method proposed in this paper. Consequently this paper verifies that when the distance among the targets is getting close, the new algorithm can improve the resolution, when CR is 0.5 , it is not only that the quality of image is satisfactory but also that it saves the bandwidth of the radar system. And in Fig. 4 for different SNR, MSE and PSNR of CS overcomes the existing RD algorithm. All these results enough verify the algorithm in this paper effectiveness. It can save storage space and transmission bandwidth in many application fields, particularly in systems having real-time imaging capability.

The CS theory has remarkable advantages of reducing sampling rate and computation, which is believed to resolve the difficult that traditional methods facing in radar applications. The new imaging method based on CS in this paper is significant in theory and in practice. The paper is still limited in theoretical analysis and simulating experiments, realizing it in engineering field should be studied further.

\section{Conclusion}

In this paper, a novel 2-D SAR imaging algorithm is proposed based on constructing measurement matrixes in range dimension via compressed sensing techniques. And the FRFT technology is used in this algorithm to enhance high resolution. This radar system randomly transmits fewer pulses in azimuth and samples fewer data than traditional systems at random intervals in range. Thereby, this method provides a new approach of receiving echo data via random sparse sampling with a significant reduction in the number of sampled data beyond the Nyquist theorem. This will directly impact A/D conversion, and has the potential to reduce the overall data rate and to simplify hardware design. The simulation results verify the validity of the proposed CS-FRFT imaging algorithm which is lower MSE and higher PSNR, less sampled data, higher resolution, stronger robustness and higher noise immunity than the traditional SAR imaging algorithm.

\section{References}

[1] Ender J H G, “On compressive sensing applied to radar,” Signal Processing, 90(2), 1402-1414, 2010.

[2] Xu, W., P. P. Huang, and Y. K. Deng, "Multi-channel SPCMB-TOPS SAR for high resolution wide-swath imaging,” Progress In Electromagnetics Research, Vol. 116, 533-551, 2011.

[3] Xie Xiao-chun and Zhang Yun-hua, "2D Radar imaging scheme based on compressive sensing technique,” Journal of Electronics \& Information Technology, 32(5), 1234-1238, 2010.

[4] Rossi, M., Haimovich, A.M. and Eldar, Y.C., "Spatial Compressive Sensing for MIMO Radar," IEEE Transactions on Signal Processing, 62(2): 419-430, 2014.

[5] Stankovic, L., Orovic, I., Stankovic, S. and Amin, M., "Compressive Sensing Based Separation of Nonstationary and Stationary Signals Overlapping in Time-Frequency,” IEEE Transactions on 
Signal Processing, 61(18): 4562-4572, 2013.

[6] J. Yang, J. Thompson, X. Huang, T. Jin, and Z. Zhou, "Random frequency SAR imaging based on compressed sensing,” IEEE Trans. Geosci. Remote Sens. 51, 983-994, 2013.

[7] W. Qiu, J. Zhou, H. Zhao, and Q. Fu, “Three-dimensional sparse turntable microwave imaging based on compressive sensing,” IEEE Geosci. Remote Sens. Lett. 12, 826-830, 2015.

[8] Huang, P., W. Xu, and W. Qi, "Two dimension digital beamforming preprocessing in multibeam scan SAR,” Progress In Electromagnetics Research, Vol. 136, 495-508, 2013.

[9] Donoho D L., “Compressive sensing,” IEEE Transactions on Information Theory, 52(4): 1289-1306, 2006.

[10]G. Oliveri, N. Anselmi, and A. Massa, "Compressive sensing imaging of non-sparse 2D scatterers by a total-variation approach within the born approximation," IEEE Trans. Antennas Propag. 62, 5157-5170, 2014.

[11]S. Li, G. Zhao, H. Li, B. Ren, W. Hu, Y. Liu, W. Yu, and H. Sun, "Nearfield radar imaging via compressive sensing,” IEEE Trans. Antennas Propag. 63, 828-833, 2015.

[12]Wu Yongjun and Huang Ye., “A Novel SAR Imaging Algorithm Based on FrFT and CS,” IEEE, 1510-1513, 2011.

[13]Sun, G.-C., Xing, M., Xia, X.-G., Yang, J., Wu, Y. and Bao, Z., “A Unified Focusing Algorithm for Several Modes of SAR Based on FrFT," IEEE Transactions on Geoscience and Remote Sensing, 51(5): 3139-3155, 2013.

[14]Xiaolong Chen, Jian Guan, Ningbo Liu and You He., "Maneuvering Target Detection via Radon-Fractional Fourier Transform-Based Long-Time Coherent Integration," IEEE Transactions on Signal Processing, 62(4): 939 - 953, 2014.

[15] Yujie Gu, Goodman, N.A. and Ashok.A., "Radar Target Profiling and Recognition Based on TSI-Optimized Compressive Sensing Kernel,” IEEE Transactions on Signal Processing, 62(12): $3194-3207,2014$. 